\title{
Cuidados com recém-nascidos alimentados por sonda gástrica
}

\author{
Care of newborns fed by gastric probe
}

Cuidado com la alimentación por tubo gástrico em recién nacido

\section{Larissa Christiny Amorim dos Santos ${ }^{1}$, Wanderson Alves Ribeiro ${ }^{2}$, Bruna Porath Azevedo Fassarella ${ }^{3}$, Keila do Carmo Neves ${ }^{4}$}

Como citar esse artigo. dos Santos, L. C. A.; Ribeiro, W. A; Fassarella, B. P. A.; Neves, K. C. Cuidados com recémnascidos alimentados por sonda gástrica. Revista Pró-UniverSUS. 2021 Jul./Dez.; 12 (2): $27-31$

\section{Resumo}

Trata-se de uma pesquisa bibliográfica de abordagem qualitativa e caráter descritivo, cujo objetivo é a reflexão sobre os cuidados com os recém-nascidos alimentados por sonda gástrica internados na Unidade de Terapia Intensiva Neonatal. Foram utilizados artigos publicados em base de dados virtuais. Para tal utilizou-se a Biblioteca Virtual de Saúde, nas bases de informações LILACS, BDENF, MEDLINE e SCIELO. A sobrevida dos recém-nascidos (RNs) tem se tornado cada vez maior em todo mundo, entretanto os RNs que nascem antes das 34 semanas de gestação apresentam dificuldade na deglutição, na sucção e até mesmo na respiração. Por isso, o benefício da alimentação enteral através de sonda gástrica tem suprido as necessidades nutricionais e alimentares. Conclui-se que o papel do enfermeiro é de fundamental importância, sendo o principal responsável pela administração da nutrição enteral (NE), além da prescrição dos cuidados de enfermagem, estabelecer o acesso enteral, por via oro/nasogástrica ou transpilórica, e assegurar o exame radiológico visando a confirmação da localização da sonda.

Palavras-chave: Terapia Intensiva Neonatal; Recém-Nascido; Enfermagem; Nutrição Enteral.

\begin{abstract}
This is bibliographic research with a qualitative approach and descriptive character, which aims to reflect on the care of newborns fed by gastric tube admitted to the Neonatal Intensive Care Unit. Articles published in a virtual database were used. For this purpose, the Virtual Health Library was used, in the LILACS, BDENF, MEDLINE and SCIELO databases, with a time frame from 2017 to 2021. The survival of newborns (NBs) has become increasing worldwide, however, newborns born before 34 weeks of gestation have difficulty in swallowing, sucking and even breathing. Therefore, the benefit of enteral feeding through a gastric tube has met nutritional and food needs. It is concluded that the role of the nurse is of fundamental importance, being the main responsible for the administration of enteral nutrition $(\mathrm{EN})$, in addition to the prescription of nursing care, establishing the enteral access, through the oral/nasogastric or transpyloric route, ensuring the examination radiological aiming at confirming the location of the probe.
\end{abstract}

Keywords: Neonatal Intensive Care; Newborn; Nursing; enteral nutrition.

\section{Afiliação dos autores:}

${ }^{1}$ Acadêmica do curso de graduação em Enfermagem da Universidade Iguaçu, Nova Iguaçú, Rio de Janeiro, Brasil. ORCID: https://orcid.org/0000-0002-9705-5811

${ }^{2}$ Enfermeiro. Mestre e Doutorando pelo Programa Acadêmico em Ciências do Cuidado em Saúde pela Escola de Enfermagem Aurora de Afonso Costa da UFF. Docente do Curso de Graduação em Enfermagem Pós-graduação da Universidade Iguaçú, , Nova Iguaçú, Rio de Janeiro, Brasil. ORCID: https://orcid.org/0000-0001-8655-3789

${ }^{3}$ Enfermeira. Mestre em Ciências Aplicadas em Saúde da Universidade Severino Sombra. Docente do Curso de Graduação em Enfermagem e Pós-graduação da UNIG, Nova Iguaçú, Rio de Janeiro, Brasil. ORCID: https://orcid.org/0000-0002-1400-4147

${ }^{4}$ Enfermeira. Pós-Graduada em Nefrologia. Mestre e Doutora em Enfermagem pela UFRJ. Docente do Curso de Graduação em Enfermagem da UNIG, Nova Iguaçú, Rio de Janeiro, Brasil. ORCID: https://orcid.org/0000-0001-6164-1336. 


\section{Resumen}

Se trata de una investigación bibliográfica con enfoque cualitativo y descriptivo, cuyo objetivo es reflexionar sobre el cuidado de los recién nacidos alimentados por sonda gástrica ingresados en una Unidad de Cuidados Intensivos Neonatales. Se utilizaron artículos publicados con base en datos virtuales. Para ello se utilizan las bases de información de la Biblioteca Virtual en Salud, LILACS, BDENF, MEDLINE y SCIELO. La supervivencia de dos recién nacidos (RN) es cada vez mayor en todo el mundo, mientras que los recién nacidos antes de la $34^{\mathrm{a}}$ semana de gestación tienen dificultades para tragar, succionar y respirar de la misma forma. Por esta razón, el beneficio de la alimentación enteral por sonda gástrica suprimió las necesidades nutricionales y nutricionales. Se concluyó que el rol del enfermero es de fundamental importancia, siendo el principal responsable de la administración de la nutrición enteral (NE), prescribiendo además dos procedimientos de atención de enfermería, estableciendo ya sea el acceso enteral, oral/nasogástrico o transpilórico, y asegurando o Exploración examen radiológico para confirmar la ubicación de la sonda.

Palabras clave: Cuidados intensivos neonatales; Recién nacido; Enfermería; nutrición enteral.

\section{Introdução}

A sobrevida dos recém-nascidos (RNs) tem se tornado cada vez maior em todo mundo, visto que mais de $10 \%$ das crianças nascem de forma prematura ${ }^{1}$. O RN prematuro (RNPT), de acordo com Organização Mundial de Saúde (OMS), são aqueles menores de 37 semanas completas de gestação, contadas a partir do primeiro dia do último período menstrual ${ }^{2}$.

Sendo considerados além da prematuridade, o baixo peso especialmente quando menor que $1500 \mathrm{~g}$, fatores de risco. Por consequência, o RNPT pode vir apresentar uma série de intercorrências, tais como, a não aceitação da alimentação por via oral (VO) e o atraso no desenvolvimento ${ }^{3}$.

Os RNs que nascem antes das 34 semanas de gestação apresentam dificuldade na deglutição, na sucção e até mesmo na respiração. Sendo a nutrição desse Rn um dos principais objetivos, o benefício da alimentação enteral através de sonda gástrica supre as necessidades nutricionais e alimentares ${ }^{4}$.

As sondas gástricas são classificadas de acordo com a sua inserção. A nasogástrica inserida por uma das narinas, e a orogástrica inserida pela cavidade oral. Em RN, é recomendado inserir a sonda pela boca, pois a inserção pela narina obstrui a respiração nasal ${ }^{5}$.

Estudos indicam que ainda existem certas dificuldades em perceber o tempo exato para iniciar a transição da alimentação gástrica para via oral, pois esses profissionais não realizam uma avaliação criteriosa que considere os aspectos e as condições do desenvolvimento com a habilidade motora, sendo o peso e a idade gestacional um dos critérios da transição ${ }^{6}$.

Cabe ressaltar que, é de responsabilidade do enfermeiro a administração da nutrição enteral (NE), além da prescrição dos cuidados de enfermagem, estabelecer o acesso enteral, por via oro/nasogástrica ou transpilórica, assegurar o exame radiológico visando a confirmação da localização da sonda, garantir que as ocorrências e dados referentes ao paciente sejam registrados de forma correta, além de orientar o paciente quanto à terapia de NE, estabelecido pelo regulamento técnico para a terapia de nutrição enteral e da norma técnica para atuação da equipe de enfermagem em terapia nutricional ${ }^{7}$.

É de suma importância do enfermeiro sempre se manter atualizado, quanto aos avanços científicos na área de neonatologia. Além de cumprerem um papel primordial na segurança e supervisão da equipe, no que diz respeito a organização da unidade e procedimentos, destacando-se a passagem de sonda orogástrica ${ }^{8}$.

O objetivo deste estudo foi refletir sobre os cuidados com o recém-nascido alimentados por sondas gástricas, a partir de artigos científicos publicados nos últimos cinco anos.

\section{Metodologia}

Trata-se de uma pesquisa bibliográfica de abordagem qualitativa e caráter descritivo. A análise foi construída a partir dos procedimentos da revisão integrativa incluindo as comparações, a busca de padrões, os dados em geral e a construção de uma cadeia lógica de evidência. Os resultados encontrados foram analisados e representam os principais dados de todo o material que possibilitou a construção de categorias.

Os dados foram coletados em base de dados virtuais. Para tal utilizou-se a Biblioteca Virtual de Saúde (BVS), na seguinte base de informação: Literatura Latino-Americana e do Caribe em Ciências da Saúde (LILACS), Literatura Internacional em Ciência da Saúde (MEDLINE), Enfermagem em foco (COFEN), Scientific Eletronic Library Online (SCIELO), dentre outros.Optou-se pelos seguintes descritores: Terapia Intensiva Neonatal; Recém-Nascido; Enfermagem; Nutrição Enteral, que se encontram nos Descritores em Ciência da Saúde (DECS). 
Estabeleceu-se então para a realização da pesquisa os critérios de inclusão: textos na íntegra e em português com abordagem da temática estabelecida e que obedecessem ao recorte temporal de 2017 a 2021 e como critérios de exclusão, os textos incompletos, textos que não abordassem a temática estabelecida e com recorte temporal inferior a 2017.

\section{Resultados e Discussão}

Subsequente a esta seleção, foi realizada uma leitura reflexiva dos artigos, onde descreveram-se os resultados encontrados nesta leitura e, ainda, uma discussão sucinta relacionada aos achados. Também foram excluídos os artigos em duplicidade e os que não atendiam aos critérios prévios.

Posterior à leitura reflexiva dos ensaios supracitados emergiram três categorias: Indicações e uso da técnica "sonda-dedo"; Sondas gástricas e sua utilização na Unidade de Terapia Intensiva Neonatal; Rede de apoio na Unidade de Terapia Intensiva Neonatal.

\section{Indicações e uso da técnica "sonda- dedo"}

O RN prematuro necessita de todo cuidado possível, sejam elas pelo cuidado da equipe de saúde ou familiar. O aleitamento materno torna-se uma estratégia necessária para uma maior criação de vínculo, afeto, proteção e nutrição para a criança e constitui a mais sensível, econômica e eficaz intervenção para redução da morbimortalidade infantil ${ }^{9}$.

Partindo desse pressuposto, o método fingerfeeding, ou como conhecido no Brasil, "sonda-dedo", consiste em uma técnica na qual o leite é ofertado por sucção ao RN prematuro através de uma sonda gástrica conectada a uma seringa e fixada no dedo mínimo enluvado com fita adesiva. Porém estudos que descrevem esta técnica ressaltam que os benefícios e seus malefícios são escassos ${ }^{10}$.

Destacando ainda esta técnica, a fim de favorecer a sucção dos RNs e como complemento de via oral. Autores avaliaram este método e concluíram que, as taxas de aleitamento materno em prematuros podem ser maiores com técnicas que corrijam a sucçãa ${ }^{11}$.

A alimentação através do dedo é citada por autores como uma opção para estimular a sucção do bebê durante a alimentação, já como consequência, exigiria maior disponibilidade de tempo por parte da equipe de saúde. Além disso, estudos mostram que RNs alimentados por sonda-dedo apresentaram menos tempo de internação além de um ganho de peso significativo $^{12}$.

Além disso, estudos aprontam a preferência dos enfermeiros pela técnica da sonda-dedo, por ser capaz de promover o perfeito desenvolvimento das competências orais, como a melhor posição da língua e o estimulo das estruturas orais de uma forma semelhante à amamentação ${ }^{13}$.

Deste modo, pude notar que as literaturas apontam que o método "sonda-dedo" complementar com as demais técnicas utilizadas na transição alimentar do prematuro, principalmente nos períodos de ausência materna e quanto à organização comportamental e estimulação da sucção durante a alimentação.

\section{Sondas gástricas e sua utilização na Unidade de Terapia Intensiva Neonatal}

A sonda gástrica é um tubo flexível, com tecnologia simples, geralmente inserida pela boca do $\mathrm{RN}$ até chegar ao estômago e é fixada externamente e muitas delas são constituídas de policloreto de polivinila $(\mathrm{PVC})^{14}$.

Sendo bastante utilizada, tendo em vista que muitos RNs não deglutem ainda com segurança devido a sua prematuridade ou devido a outras causas. Tornase necessário se atentar para os erros cometidos durante essa administração. Utilizadas muita das vezes para suporte nu-tricional, administração de medicamentos e/ ou descompressãogástrica ${ }^{15}$.

Na UTI Neonatal, o posicionamento correto de sondas e cateteres evitam ou minimizam complicações. No entanto, apesar da radiografia ser o principal método de confirmação do posicionamento das sondas gástricas, ela pode expor as crianças à radiação. A localização por ausculta de injeção de ar também pode ser falha se a sonda se encontrar na terceira parte da região traqueobrônquica ${ }^{16}$.

O padrão-ouro utilizada para verificar a localização da sonda é a radiografia abdominal, mas em neonatologia, não é possível expor o $\mathrm{RN}$ às múltiplas radiografias para esta finalidade (como dito no parágrafo anterior), por isso, o mais indicado é a visualização em tempo real ${ }^{17}$.

A avaliação do aspirado do resíduo gástrico (RG) também é utilizada, no entanto pode haver dificuldade de identificação visual desse aspirado em UTINeonatal, pois o resíduo salivar e o leitoso podem ser confundidos com secreção traqueal ${ }^{18}$.

Existem dados que mostram frequentes erros no posicionamento de sondas em crianças, e esses fatores de risco associados à má colocação incluem a diminuição do nível de consciência, distensão abdominal, vômitos, e lesões graves e até mesmo morte ${ }^{19}$

Em vista dos possíveis resultados catastróficos que podem ocorrer por uma sonda mal posicionada, se torna necessária a seriedade dos profissionais e 
a importância de estratégias para as Boas Práticas com vistas à segurança do paciente, evitando erros e mitigando eventos adversos.

\section{Rede de apoio na Unidade de Terapia Intensiva Neonatal}

A equipe de uma Unidade de Terapia Intensiva Neonatal é formada por neonatologistas, fisioterapeutas, fonoaudiólogos, enfermeiro gerente, enfermeiro assistencial, auxiliar e técnico de enfermagem. Tendo o enfermeiro destaque, por lidar com situações emocionais difíceis, com a fragilidade de um RN extremo, com a morte, sentimentos de ansiedade e insegurança por parte dos familiares ${ }^{20}$.

O cuidado de enfermagem se mostra cada vez mais essencial, tendo na sua assistência o papel de educador, cabe ao enfermeiro orientar as mães, ouvir suas dúvidas e as esclarecer da melhor forma possível, além de orientar sobre a importância do contato pele a pele nesse momento ${ }^{21}$.

Esse contato pele a pele desde cedo, auxilia na criação de laços afetivos, formação de vínculo, melhoria dos sistemas imunes e respiratórios, alívio da dor e termorregulação; enfatizando que os benefícios ultrapassam o cuidado intra-hospitalar através do empoderamento familiar ${ }^{22}$.

Foi evidenciado que, mães de $\mathrm{RN}$ internados na UTI-Neonatal se sentem valorizadas quando podem contribuir com o processo de alimentação do bebê. Através dessa perspectiva, constrói-se um processo de cuidado, obtendo bons resultados nas ações de enfermagem que dependem do bom relacionamento, preservando a singularidade e a individualidade da criança e de seus pais ${ }^{23}$.

A rede de apoio pode apresentar ansiedade e fatores estressores, como por exemplo, sobrecarregar os pais com perguntas ou reações emocionais exacerbadas, assim como fantasias acerca do diagnóstico, prognóstico e tempo de internação do $\mathrm{RN}$, até mesmo pode-se questionar a competência dos pais para lidar com o processo de cuidado de seus filhos ${ }^{24}$.

Cabe refletir que a angústia está muita das vezes ligada a distância de seus entes queridos, o medo da perda do RN internado na UTI, e em muitos casos, pela falta de condição financeira para ir e vir do hospital. Cabe ressaltar ainda que o profissional enfermeiro se destaca e que por lidar com a família desse RN internado na UTI, necessita de um olhar cuidadoso, dando apoio e incentivando para que essa família mesmo com todas as dificuldades no percurso da internação, siga firme ao lado do seu filho ${ }^{25}$.

\section{Conclusão}

Conclui-se que, o enfermeiro é um profissional que possui em sua essência o cuidado ao ser humano em todas as suas necessidades, assistindo-o individualmente, no contexto familiar e no da comunidade. E sendo um integrante da equipe de saúde que atua na UTINeonatal, necessita de conhecimentos técnicos e científicos sobre as suas atribuições específicas e privativas, bem como ser capacitado para prestar uma assistência de qualidade a esta clientela e sua família.

No entanto, faltam evidências que comprovem as melhores formas de administração por sonda gástrica, quando iniciar a dieta, qual o volume mínimo, como avaliar a progressão da dieta, qual o volume de dieta que se pode considerar como dieta enteral total e as técnicas de transição da sonda gástrica para a via oral. Através desse estudo, espera-se contribuir para melhoria das ações de enfermagem frente aos cuidados com o RN em uma UTI Neonatal, além de estimular a elaboração de outros estudos relacionados a esta temática.

\section{Referências}

1. Nascimento, J, Santos IMM, Silva LJ. Cuidados com recém-nascidos alimentados por sonda gástrica: conceitos e práticas. Texto Contexto Enferm [Internet]. 2019;28:e20170242.

2. Silva RMM, Zilly A, Ferreira H, Pancieri L, Pina JC, Mello DF. Fatores relacionados ao tempo de hospitalização e óbito de recém-nascidos prematuros. Rev Esc Enferm USP. 2021;55:e03704.

3. Medeiros AMC, Almeida DMS, Meneses MO, Sá TPL, Barreto IDC. Impacto da intervenção fonoaudiológica na introdução de dieta via oral em recém-nascidos de risco. Audiol Commun Res.2020;25:e2377.

4. Silveira BL, Santos RCS, Araújo MGS, Lacerda GAN, Mascarenhas MLVC, Guedes BLS. Correlação de dados de antropometria facial de recémnascidos prematuros tardios e prontidão para alimentação oral.Rev Bras Enferm. 2021;74(5):e20201120.

5. AO, TRANSIÇÃO DA NUTRIÇÃO ENTERAL. 4.1 Técnica de transição direto da sonda para seio materno (SM). Disponível em: https://www.gov.br/ebserh/pt-br/hospitais-universitarios/regiao-nordeste/ hupaa-ufal/acesso-a-informacao/procedimento-operacional-padrao/ fonoaudiologia/pop 05 transicao-nutricao-enteral-ao-aleitamento-maternoem-recem-nascidos-unidade-neonatal_versao_final_sem_assinaturas.pdf.

6. Acioli DMN, dos Santos AAP, Lúcio IML, Ferreira ALC. Transição alimentar do Recém-nascido prematuro para introdução do aleitamento materno. Brazilian Journal Devopelopment. 2020. 6(12):103382-103393.

7. Manzoli BS, Zamberlan P, Leite AGZ, Reis APA. COVID-19 em pediatria: sugestões para o manejo nutricional. BRASPEN J. 2020; 35 (2):107-13.

8. Silva TL, de Oliveira AEC, de Carvalho JO, de Paiva EP, Thofehrn MB, Borel MGC. Relato de experiência sobre aplicação do método mãe canguru em uma Unidade de Terapia Intensiva Neonatal. Brazilian Journal of Health Review. 2020;3(4):8767-74.

9. Moreira CM, Cavalcante-Silva RP, Fujinaga CI, Marson F. Comparação entre o método sonda-dedo versus copo na transição alimentar do recém-nascido prematuro. Jornal de Pediatria. 2017; 93(6):585-91.

10. Sousa CM. Performance alimentar do recém-nascido pré-termo: Da alimentação por sonda até à autonomia. 2021. ESEP - Escola Superior de Enfermagem do Porto ESEP - Dissertações de Mestrado. 
11. Nunes JA, Bianchini EMG, Cunha MC. Saturação de oxigênio e frequência cardíaca em prematuros: comparação entre as técnicas de copo e sonda-dedo. CoDAS. Sociedade Brasileira de Fonoaudiologia. 2019;31(6):e20180221

12. Medeiros MLO. Métodos alternativos para administração da dieta ao recém-nascido: revisão integrativa. 2021. Trabalho de Conclusão de Curso. Universidade Federal do Rio Grande do Norte.

13. Brantes ALG, Curado MAS. Percepção dos enfermeiros sobre a alimentação do recém-nascido pré-termo. Texto \& Contexto-Enfermagem. $2021 ; 30$.

14. Soares LS. Avaliação da retirada não eletiva dos tubos orogástricos em recém-nascidos prematuros. 2018. Dissertação de Mestrado.

15. da Silva HR, Ferreira LP, Fernandes MTC, Dellanhese APF. Métodos alternativos de verificação do posicionamento de sonda gástrica em crianças. Saúde Coletiva (Barueri), 2020; 10(59): 4326-4337.

16. Vieira SA, de Araújo N, da Silva AR, de Souza DP. Análise da eficácia do método canguru: recém-nascido de baixo peso. Revista Recien-Revista Científica de Enfermagem. 2020;10(32):44-52.

17. André RR, Mendes CQDS, Avelar AFM, Balieiro MMFG. Posicionamento de sonda enteral em neonatos segundo técnica modificada de mensuração. Acta Paulista de Enfermagem. 2017; 30:590-97.

18. Soares LS, Silva GRF, Machado RS. Evidências científicas sobre uso e cuidados de enfermagem com tubos orogástricos em neonatos prematuros. Rev. Soc. Bras. Enferm. Ped. 2017;17(1):37-42.

19. Sousa CM. Performance alimentar do recém-nascido pré-termo: Da alimentação por sonda até à autonomia. 2021. ESEP - Escola Superior de Enfermagem do Porto ESEP - Dissertações de Mestrado.

20. Batista CDM, Monteiro JC, Pinheiro VR, Soares TB, de Lima FC, Nascimento MHM, Ueno TMRL. Diagnósticos e cuidados de enfermagem ao recém-nascido prematuro em Unidade de Terapia Intensiva Neonatal. Revista Eletrônica Acervo Saúde. 2019;(35):e1593-e1593.

21. Gimenez VCA. Análise de incidentes relacionados a carga de trabalho de enfermagem em unidade de terapia intensiva neonatal. 2020. Faculdade de Medicina (FMB) - Botucatu Pós-Graduação em Enfermagem Dissertações Enfermagem - FMB.

22. Costa DG, de Castro HO, Passos RC, Lopes PA, Firmino VHN. A percepção da equipe de enfermagem sobre o método canguru. Revista IberoAmericana de Humanidades, Ciências e Educação. 2021;7(9): 451-468.

23. Lima SES. Maternidade prematura, apoio social e necessidades de mães de neonatos hospitalizados na Unidade de Terapia Intensiva Neonatal. MS thesis. Universidade Federal do Rio Grande do Norte, 2020.

24. Taurisano AAA, Enumo SRF, Prebianchi HB, Andrade ALM. Estresse e satisfação de pais com o atendimento em unidade de terapia intensiva neonatal. Interação em Psicologia. 2020; 24(2).

25. de Oliveira MP, Rodrigues AC, de Souza Corrêa BS, dos Santos Dias CT, da Silva Bomfim VVB, Mascarenhas BB, Bastos PRL. Fatores que dificultam o aleitamento materno na Unidade de Terapia Intensiva Neonatal. Pesquisa, Sociedade e Desenvolvimento. 2021; 10(8): e39010817190e39010817190. 